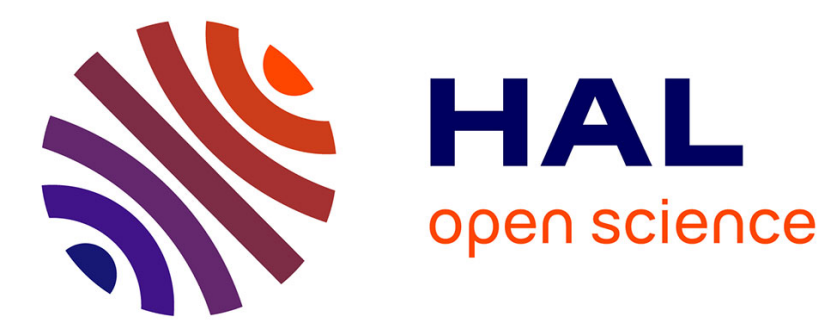

\title{
L'enfant dans l'espace commercial : éléments pour une mise en perspective
}

\author{
Valérie-Inès de La Ville
}

\section{To cite this version:}

Valérie-Inès de La Ville. L'enfant dans l'espace commercial: éléments pour une mise en perspective. Revue management \& avenir, 2009, 21, pp.157-171. 10.3917/mav.021.0157 . hal-01844025

\section{HAL Id: hal-01844025 \\ https://hal.science/hal-01844025}

Submitted on 14 Mar 2019

HAL is a multi-disciplinary open access archive for the deposit and dissemination of scientific research documents, whether they are published or not. The documents may come from teaching and research institutions in France or abroad, or from public or private research centers.
L'archive ouverte pluridisciplinaire HAL, est destinée au dépôt et à la diffusion de documents scientifiques de niveau recherche, publiés ou non, émanant des établissements d'enseignement et de recherche français ou étrangers, des laboratoires publics ou privés. 


\title{
L'enfant dans l'espace commercial : éléments pour une mise en perspective...
}

\author{
The child in the High Street store: \\ elements to put things into perspective...
}

\author{
Valérie-Inés de La Ville \\ Professeur Université de Clermont 1 \\ Centre Européen des Produits de l'Enfant et laboratoire CEREGE de l'Université de Poitiers ${ }^{1}$ \\ delaville@iae.univ-poitiers.fr
}

Résumé : Le thème de l'enfant ( 0 - 12 ans) dans l'espace commercial est peu traité dans les recherches sur la distribution et dans celles portant sur les jeunes consommateurs. Or, depuis les années 1990, des enseignes spécialistes de l'enfant se développent en testant et mettant en œuvre des innovations empiriques. Cet article offre une première approche de ce champ et s'interroge sur les enjeux propres aux enseignes de distribution qui s'affirment spécialistes de l'enfant.

\begin{abstract}
The theme of the child (0 - 12 years) in the High Street store is hardly touched upon in research studies on distribution and young consumers. Yet since the 1990's shops specializing in children's products have been developing by testing and implementing empirical innovations. This article offers a first glimpse on this subject and examines the distinctive characteristics of distributional outlets who claim to be specialists in children's products.
\end{abstract}

\section{Introduction : Des pratiques professionnelles inventives qui appellent un effort de recherche}

Le thème de l'enfant dans l'espace commercial est assez peu traité dans les manuels de référence sur les univers de la distribution généraliste ou spécialisée (Jallais, Orsoni \& Fady 1987 ; Filser \& Jallais 1988 ; Volle 2000 ; Cliquet, Fady \& Basset 2002, Benoun \& HélièsHassid 2003, Gallouj 2007, Mouton \& Paris 2007...) $)^{2}$. En ce qui concerne les GSA, les enfants constituent depuis plusieurs années une cible privilégiée des MDD comme Auchan avec la marque « Rik et Rok » ou Carrefour avec la marque « Genius ». L'objectif stratégique pour les distributeurs généralistes étant de dépasser $50 \%$ du chiffre d'affaires avec des marques propres d'ici 2010, l'enfant va clairement se réaffirmer comme une cible prioritaire. En effet, savoir recruter des enfants avec des marques qui leur dont destinées est un moyen à court terme de fidéliser les parents et à long terme de créer une relation de confiance avec les futurs consommateurs adultes. A partir des années 1990, un certain nombre de chaînes de

\footnotetext{
${ }^{1}$ Cette recherche bénéficie du soutien de l'Agence Nationale de la Recherche dans le cadre du Programme National de Recherche sur l'Alimentation - projet «Ludo-Aliment » coordonné par l'auteur.

${ }^{2}$ Dans les ouvrages qui contiennent un index, il n'ya pas d'entrée « Enfant ».
} 
magasins spécialisés sur les produits de l'enfant se sont développées (Héliès-Hassid M.L. 2007). Il s'agit par exemple des magasins «Imaginarium» en Espagne ou de la «Fnac Junior » en France, qui ont réellement été des enseignes pionnières, ayant la volonté de remettre en cause un certain nombre de principes classiques d'organisation et de gestion d'un magasin (format, assortiment, mobilier, circulation, merchandising, modalités de mise en contact avec les produits, etc.) afin d'accueillir l'enfant dans des conditions qualitatives. Ces espaces commerciaux dédiés à l'enfant ont permis aux parents comme aux enfants de découvrir de nouvelles catégories de produits demandant un conseil spécialisé, tels que les produits «ludo-éducatifs" en pleine croissance à l'époque. Depuis, dans des domaines très divers de l'équipement de la personne, des loisirs ou même de la distribution alimentaire, d'autres enseignes ont poursuivi ce mouvement et se sont déclarées spécialistes de l'enfant : « Apache », «Orchestra-Kazibao », «Okaïdi », « Kidfresh »... Les pratiques innovantes des enseignes ayant créé un concept de distribution dédié à l'enfant - qui expriment une grande créativité empirique - constituent un thème qui mérite par conséquent d'être exploité et approfondi par les chercheurs en marketing. Cet article propose une première approche de ce champ, sans aucune prétention à l'exhaustivité, dans le but d'illustrer quelques pratiques professionnelles innovantes et d'interroger ce que révèle la relation qui s'instaure entre les enfants et leurs parents et les enseignes de distribution qui s'affirment spécialistes de l'enfant.

\section{L'enfant et l'espace commercial : pluralité de rôles et de contextes d'interaction}

L'ensemble des chercheurs intéressés par le sujet s'accorde sur le fait que pour aborder le thème de l'enfant dans l'espace commercial, il convient de distinguer une pluralité de rôles de l'enfant, qui dépendent des situations, de son âge, de ses capacités cognitives et relationnelles ainsi que de la façon dont l'espace est aménagé pour l'accueillir. Nous reformulons les enjeux liés à quatre rôles clés que peut jouer l'enfant selon la nature de sa relation à l'espace commercial en nous fondant sur la distinction apportée par M.L. Héliès-Hassid (2007)

\section{1 - Différentes modalités du rapport de l'enfant à l'espace commercial}

- La décision sur le lieu de vente échappe à l'enfant, mais il peut l'influencer : l'enfant reste à la maison, mais il reçoit des produits qui proviennent de l'espace commercial, où ses parents ont acquis pour lui un certain nombre de produits. Ainsi, sans être présent physiquement dans l'espace commercial, l'enfant entretient déjà une relation avec celui-ci, même si cette relation est indirecte et se traduit à la fois par le choix de ses parents et par l'ensemble des produits que ces derniers rapportent au foyer familial. Même dans ces conditions distanciées de l'espace commercial, l'enfant s'initie progressivement à travers le produit au vocabulaire de l'univers commercial (packaging, marques, prix, rapport qualité / prix, logos, personnages de marque, etc.).

- L'enfant peut intervenir directement dans la décision de l'achat en étant présent aux côtés de ses parents, grands-parents ou grands frères dans les magasins qui ciblent la famille. Il va donc être accompagné sur le lieu de vente, y découvrir la présentation en linéaire des produits qu'il reconnait et avoir l'opportunité de s'intéresser à l'ensemble de l'offre qui lui est proposée. Il va s'initier aux gestes et réflexes incorporés par le consommateur sur le lieu de vente (guidage du chariot, déambulation, compréhension de la signalétique, agencement des rayons, affichage des prix, informations portées sur le « facing » des produits, organisation des linéaires, promotions en tête de gondoles, 
écoute de la musique d'ambiance, gestion du temps, etc.). Particulièrement curieux, l'enfant va attirer le regard de ses accompagnateurs sur les nouveaux produits, sur les opérations promotionnelles liées à une licence, sur les produits proposant un cadeau qui l'intéresse, etc. Du fait de ces nouveaux éléments qu'il va porter à la connaissance des adultes, qui eux suivent bon nombre de routines pour faire leurs courses, l'enfant va être en mesure de négocier sur certain nombre de points afin d'obtenir le produit qu'il souhaite tester ou avoir. Mais la nouveauté du produit ou l'intérêt du cadeau ne suffisent pas, et l'enfant devra également justifier sa demande par une rhétorique adaptée à la fois aux circonstances et à la nature du produit («j'ai été sage »; «c'est bon pour les enfants »; «tous mes copains en ont déjà »; «c'est bientôt mon anniversaire », etc.)

- L'enfant est considéré comme complètement autonome, et il évolue dans des magasins qui ont été entièrement conçus pour lui, pour qu'il puisse y passer du temps, y déambuler à son aise et y découvrir à son rythme différents produits. Cette tendance s'est affirmée au début des années 1990 avec la création d'enseignes comme «Imaginarium », «La Fnac Junior» et «Apache », etc. Ces enseignes pionnières se sont différenciées grâce à une sélection très stricte de produits dits «ludo-éducatifs » car adaptés aux capacités socio-cognitives des enfants ainsi que par la présence vendeurs spécialisés - chez «Imaginarium», ces vendeurs arborent le titre de «jouégologues»- qui orientent les parents sur le meilleur choix pour le développement de leur progéniture. En outre, de nombreux ateliers et animations sont

\begin{tabular}{|l|}
\hline \multicolumn{1}{|c|}{ IMAGINARIUM } \\
Création en 1992 en Espagne \\
Cible 0 - 12 ans \\
1200 références en moyenne \\
Surface moyenne 60 à $80 \mathrm{~m} 2$ \\
Ticket moyen $20 €$ \\
Réseau de 340 magasins dans \\
28 pays en 2008 \\
\hline
\end{tabular}

\author{
FNAC JUNIOR EVEIL \& JEUX \\ Création en 1997 en France \\ Cible 0 - 12 ans \\ 6000 références en moyenne \\ Surface moyenne $250 \mathrm{~m} 2$ \\ Ticket moyen $20 €$ \\ Réseau de 40 magasins en 2008 et \\ vente en ligne : 1 million de clients
}

organisés, soit à titre gracieux, soit à titre payant comme les fêtes d'anniversaires. L'agencement du magasin et son mobilier sont conçus pour permettre aux enfants de prendre les produits, de commencer à les utiliser, de jouer avec. Les univers de consommation sont organisés et théâtralisés selon les centres d'intérêt des enfants : confiserie, jeux créatifs, musique, maquillage, déguisements, jeux vidéo, etc. Dans ce type de magasins, l'enfant évolue dans un environnement « ludique sécurisé »: la sélection faite sur les produits écarte les produits controversés tels des jeux vidéo violents par exemple. Toutefois, si l'enfant circule librement et de façon autonome en apparence, son autonomie est relative car il évolue dans un environnement fortement contraint par une norme adulte portant sur ce qu'est une pratique ludique socialement acceptable et sur ce qui constitue des enjeux essentiels pour son développement sociocognitif.

L'enfant est un vecteur d'innovation dans la cellule familiale car il contribue à transformer les modalités selon lesquelles ses propres parents achètent les produits. Etant curieux et ouvert à un certain nombre de nouvelles techniques de commercialisation, l'enfant peut inciter ses parents ou ses pairs à faire des recherches 
d'informations sur Internet sur différents produits, et même éventuellement les guider dans leurs achats sur Internet. Il ne faut surtout pas sous-évaluer ce rôle de l'enfant dans la cellule familiale car, même s'il n'est pas en mesure d'acheter lui-même les produits, il dispose parfois d'une grande capacité d'influence pour orienter les recherches de ses proches (amis, fratrie, parents et grands-parents) et pour signaler les nouveautés dont il a connaissance sur les sites ludiques que les marques lui proposent (Moore 2006) ou les sites de jeux qu'il fréquente assidûment (Fielder et al. 2007).

\section{2 - Accueillir l'enfant sur le lieu de vente : quelques éléments de « retail marketing »}

Quelles sont les techniques de «retail marketing » qui sont appliquées pour accueillir l'enfant et ses parents sur le lieu de vente et susciter sa participation au choix des produits qui le concernent? Nous illustrons certaines de ces techniques avec le cas du groupe «Ludendo » ci-après :

\section{Encadré $\mathbf{N}^{\circ} 1$ : Le concept LUDENDO VILLAGE}

\section{Un groupe de distribution spécialiste de l'enfant}

Le groupe «Ludendo » dont l'origine remonte à 1977, a réalisé en 2007 un chiffre d'affaires HT de $300 \mathrm{M} €$ au travers de 350 magasins détenus en propre. Groupe à vocation universelle pour l'enfant, «Ludendo » gère 12 enseignes correspondant à des métiers dfférents. Le métier historique du jouet reste l'activité principale du groupe, parallèlement à des métiers complémentaires au jouet, tels que la puériculture, le livre jeunesse, le jeu vidéo, les cadeaux, et la décoration de la chambre d'enfant.

\section{Effets de synergies dans un groupe multi-métier, multi-enseigne, multi-format}

Ludendo est un groupe multi-formats, avec une solution pour tous types d'implantations, qui possède des magasins en centre-ville, en centre commercial, en périphérie, sur des surfaces de 300 à $4000 \mathrm{~m}^{2}$. Ainsi, le groupe gère un réseau très divers et particulièrement complexe de formats. En outre, le groupe est implanté dans différents pays, en France, en Belgique, en Suisse, en Espagne et au Maroc. Dans cette configuration, un enjeu clé consiste à créer des effets de synergies entre enseignes. Dans ce but, la symbolique du groupe a été retravaillée : la double main signifie à la fois l'adulte et l'enfant, la transmission, l'accompagnement et le partage. Et un concept de magasin innovant a été lancé pour refléter les valeurs de la marque «Ludendo » créée il y a deux ans pour donner une identité au groupe.

\section{Le concept « Ludendo Village »}

«Ludendo Village » est conçu comme un lieu universel, pour tous les enfants du monde, qui exprime des valeurs fortes et n'est pas simplement sur un territoire marchand, mais d'abord un territoire de valeurs, celles du groupe. Le premier "Ludendo Village» a ouvert en novembre 2006 dans le centre commercial Val d'Europe, à l'Est de Paris. «Ludendo Village » c'est un site de $4000 \mathrm{~m}^{2}$ au total, qui fédère tous les métiers, à savoir le jouet, avec la Grande Récré, B comme BéBé, pour la puériculture, Cado Déko, pour tout ce qui concerne la décoration de la chambre, et Lire et Grandir, pour le livre de jeunesse et même la confiserie avec la Farandole des Gourmands... 


\begin{abstract}
Assortiment et extension de la cible
Au niveau de chaque enseigne, nous proposons des concepts purs, à la fois très signifiants et impactants dans l'esprit du client. L'enseigne «Lire et Grandir »- le livre jeunesse, pour les 0-15 ans - ne peut offrir tous les livres car cela conduirait à un référencement trop large, mais toutes les catégories de livres sont présentes. Et dans chacune de ces catégories, nous sélectionnons les livres qui apportent quelque chose à l'enfant, soit parce que c'est dans l'air du temps, soit parce que cela va contribuer à son épanouissement et à son éducation. A l'intérieur de "Lire et Grandir », l'offre de livres est complétée par des produits éducatifs, créatifs, scientifiques, mais nous n'exposons que trois ou quatre jouets. Nous avons un véritable rôle d'hyper-sélection, de façon à proposer une offre qui soit très pointue et en phase avec le positionnement et la promesse de la marque. "Ludendo Village " propose ainsi une palette de produits qui va permettre d'intervenir auprès des enfants de 0 à 15 ans, ce qui double notre cible, puisque dans un magasin de jouets, la cible est constituée par les enfants de 0 à 7 ans. Le concept permet de coupler une démarche d'attribution de sens avec une démarche marchande. Pour les différentes enseignes et en particulier pour le jouet, il nous faut à la fois séduire les enfants et rassurer les parents.
\end{abstract}

\title{
La place du village et théâtralisation des univers
}

L'élément clé du concept «Ludendo Village » est l'aménagement d'une une grande zone d'animation centrale qui fait le lien entre les différentes enseignes : les clients peuvent s'y promener comme s'ils étaient dans un vrai village, pouvant passer d'un magasin à l'autre, au gré de leur temps disponible et de leurs envies. Le client se trouve donc dans un petit village, où il peut se promener, où chaque magasin est constitué comme une boutique de centre-ville, avec une entrée, une vitrine animée, qui est renouvelée très régulièrement. Le client va venir soit pour un seul de ces magasins, soit pour l'ensemble du village, en fonction de ses besoins. Nous apportons un soin particulier à la théâtralisation des têtes de gondole. Il faut savoir que dans l'univers du jouet, la tête de gondole n'a rien à voir avec la logique qui prévaut en supermarché : l'objectif n'est pas de faire de la vente additionnelle dans une période courte, mais d'offrir un prolongement du rayon par une mise en avant féérique.

\section{Animations et expérience de magasinage}

L'ensemble des animations proposées sur la place du village, appelées « récréactivités », sont gratuites, ce qui crée une autre relation avec le client, puisqu'on lui propose autre chose qu'une simple relation marchande : il peut venir passer un bon moment, venir se détendre, musarder... C'est très attractif pour les enfants. Les animations sont réalisées à la fois par une équipe interne de quatre personnes dédiée à cet espace et par des prestataires extérieurs sur des thématiques techniques comme le cirque, etc. Pendant que les enfants participent aux animations, une aire de repos et d'attente a été aménagée pour les parents et les grands-parents, ce qui leur permet de patienter sans s'impatienter. Audelà des achats programmés ou des achats d'impulsion, les clients vont pouvoir passer un bon moment, se divertir, et donc partir avec un bon souvenir, une bonne image du groupe. C'est important car c'est une façon de fidéliser et c'est aussi une façon de recruter.

Source: extraits de la présentation faite par Monsieur Franck Mathais, Directeur de la communication du groupe « Ludendo » au X ${ }^{\circ}$ Colloque Etienne THIL en Octobre 2007

Sans souci d'exhaustivité, nous pouvons souligner que les techniques de « retail marketing » mobilisées par les enseignes spécialistes de l'enfant comprennent : 
- Un assortiment volontairement limité résultant d'une sélection drastique de produits en fonction de leur originalité mais aussi de l'adéquation des fonctionnalités qu'ils proposent aux différents stades de développement socio-cognitif de l'enfant. Cette hyper-sélection est au cœur du métier de ces enseignes et suppose de développer une compétence d'acheteur spécialiste de l'enfant bien au-delà des seules normes techniques et du cadre législatif en vigueur selon les matériaux entrant dans la composition du produit (plastique, textile, jouet électronique, peintures, etc.). Les « category killers » comme «Toys R Us » pratiquent une sélection plus segmentée qui a pour but d'offrir une large gamme de produits par catégorie de façon à couvrir dans le même point de vente l'ensemble de la demande ou bien de démultiplier les enseignes qui n'ont d'autre choix alors que de se sur-pécialiser comme par exemple «Babies R Us ».

- Une grande clarté dans la définition des univers de façon à faciliter l'orientation des parents et les enfants vers les catégories de produits qu'ils recherchent. En général, deux types de catégorisation sont privilégiés : soit par tranche d'âge, ce qui est naturel chez les spécialistes de l'équipement de la personne, mais qui pour des produits culturels et éducatifs est moins intuitif et suppose de justifier ce choix vis-à-vis du client (Szymansky 2002) ; soit par catégorie d'activité ludique, ce qui confronte parfois les responsables marketing à des choix cornéliens pour situer des innovations telles que les DVD interactifs ludiques qui, quand ils sont apparus, ne pouvaient être classés ni parmi les DVD ni parmi les jeux vidéo ou les CDRoms ludo-éducatifs puisqu'ils ne s'utilisent pas avec un ordinateur... La signalétique doit clairement révéler l'organisation du magasin et être pensée pour permettre une double lecture : principalement iconographique pour l'enfant (Cowles 2002) et davantage textuelle pour l'adulte.

- Un effort d'aménagement de l'espace selon plusieurs principes parfois contradictoires entre lesquels il faut arbitrer. Le marketing sensoriel du point de vente (Daucé \& Rieunier 2002, Weiner 2004) devient un enjeu majeur mais particulièrement complexe car il doit prendre en compte l'accueil simultané des parents et des enfants sur le lieu de vente. Des enseignes comme «Imaginarium» ou «Apache» ont choisi de juxtaposer deux portes, l'une réservée aux enfants et l'autre aux adultes. Les magasins «Orchestra » ont élargi les allées pour que les mères puissent circuler dans les rayons avec leur enfant confortablement installé dans sa poussette et ont aménagé un espace détente pour que les pères visionnent sur un écran des événements sportifs en attendant que l'essayage des habits se termine. Dans des magasins de mode ciblant des adolescentes, la surface des cabines d'essayage a été doublée de façon à ce qu'elles partagent ce moment de complicité entre amies. Les lieux de vente deviennent des lieux accueillant toute la famille, ce qui suppose d'inclure des espaces pour changer bébé ou pour faire réchauffer son biberon, ou encore de mettre en place des animations pour occuper les grands frères...et redonner de la liberté aux parents ou grands-parents pour déambuler à leur rythme.

- Une théâtralisation des univers accompagnée de services spécifiques qui peut être permanente selon les catégories de produits mis en scène ou plutôt temporaire car liée à des opérations promotionnelles. Féérique du point de vue de l'enfant, elle permet également de varier les thématiques et donc de créer un lien entre le lieu de vente et l'actualité des cultures enfantines, de ce qui intéresse les enfants et dont ils parlent entre eux : dessins animés, séries TV, films, jeux vidéo, etc. Cela implique de faire de 
la veille sur des propriétés émergentes qui viennent de différents secteurs culturels, et de savoir utiliser le licensing à des degrés très différents : concevoir des lignes de produits spécifiques arborant la licence, ou au contraire, faire des opérations promotionnelles ponctuelles pour animer le magasin. Car cette théâtralisation corrélée à des animations - organisation d'ateliers comme chez «Cultura» ou de fêtes d'anniversaires par «Apache» - constitue une voie de recrutement de nouveaux enfants qui demandent à leurs parents de les inscrire pour passer un moment entre copains.

- Une capacité à valoriser les produits sélectionnés auprès des parents à travers notamment des fiches techniques, présentes sur le lieu de vente comme sur le site web, expliquant de façon détaillée le bénéfice - hédonique mais aussi développemental que va tirer l'enfant de l'usage du produit. Cette rhétorique marketing s'appuie sur des connaissances en psychologie récentes et largement diffusées par les médias, comme les magazines destinés aux parents ou les nombreux sites d'accompagnement à la parentalité.

- Une capacité à valoriser les parents eux-mêmes, à les rassurer sur la pertinence du choix qui les porte vers des produits plus onéreux mais qui ont été conçus pour accompagner et susciter le développement harmonieux de leur enfant. De ce fait, le personnel de vente doit parvenir à reformuler, grâce à des termes simples ou à des métaphores adaptées, non seulement le bénéfice que retirera l'enfant, mais également celui que ressentira l'adulte dans son rôle de parent ou de grand-parent attentif.

Ainsi, l'aménagement du lieu de vente offre des médiations non seulement d'ordre matériel mais aussi de nature symbolique qui contribuent à façonner l'expérience de consommation de l'enfant tout en valorisant les parents et les grands-parents dans leur rôle d'éducateur attentif et soucieux du bien-être de leur progéniture. L'organisation du lieu de vente pour y accueillir toute la famille, la théâtralisation de l'espace pour attirer et séduire l'enfant, ainsi que l'apport d'un conseil fondé sur une argumentation du bénéfice pour le développement de l'enfant apparaissent comme les éléments les plus caractéristiques du savoir faire empirique développé par ces enseignes spécialistes de l'enfant, tant dans les magasins que sur les sites de vente en ligne. A la fois pour les parents et les enfants, les techniques de « retail marketing » rendent l'expérience conjointe des courses plus ludique et plus agréable en limitant les occasions de conflits et en valorisant l'activité de chacun dans l'espace commercial.

\section{2 - L'espace commercial dédié à l'enfant : susciter et accompagner la participation de l'enfant aux activités de consommation}

Cette deuxième partie propose d'élargir la réflexion pour montrer que ces innovations qui conduisent à concevoir des espaces commerciaux entièrement dédiés à l'enfant constituent l'aboutissement d'un mouvement plus profond par lequel les techniques marketing contribuent à la fois à susciter, accompagner et légitimer la transformation de la participation de l'enfant aux activités de consommation dans la société contemporaine. Cette réflexion s'appuie sur des travaux qui soulignent l'importance de resituer ce type d'innovations dans une perspective historique. 


\section{1 - Les enseignements d'une mise en perspective historique...}

L'accueil de l'enfant dans l'espace commercial ne constitue pas une pratique professionnelle nouvelle. M.L. Héliès-Hassid (2000) rappelle qu'Emile Zola décrit de façon détaillée dans son roman « $\mathrm{Au}$ Bonheur des Dames» la politique marketing du grand magasin « $\mathrm{Au}$ Bon Marché » créé en 1852 par Aristide Boucicaut. Se fondant à a fois sur les notes prises lors de l'enquête ethnographique menée par Zola pour réunir les éléments nécessaires à la préparation du roman, et sur le roman, lui-même, M.L. Héliès-Hassid souligne que l'importance de prendre en compte l'enfant pour fidéliser la mère constituait déjà une technique marketing clairement établie. Bien qu'ils ne soient pas décrits en détail dans le roman, les dispositifs matériels et symboliques mobilisés dans ce but sont clairement évoqués : création de rayons pour petits garçons et fillettes, cadeaux d'images et ballons arborant le nom du magasin aux enfants...

C'est Dan Cook (2004) qui, dans son analyse de l'évolution des pratiques commerciales de l'industrie textile aux Etats-Unis depuis la fin du XIXème siècle, décrit avec minutie les dispositifs marketing mobilisés par les fabricants et les distributeurs d'articles de mode et d'habillement. Ce travail rigoureux met en œuvre une démarche de triangulation des données historiques par un croisement permanent entre des sources variées : catalogues de fabricants, catalogues de distributeurs, revues professionnelles destinées aux distributeurs mais également, revues adressées aux parents de milieux sociaux privilégiés discutant et promouvant de nouvelles approches éducatives. Ainsi Dan Cook rappelle que dès 1910, sur la même idée que celle qui animait Aristide Boucicaut, de grands magasins et certains hotels avaient aménagé des pièces de jeux conçues et aménagées en fonction des goûts attribués aux enfants pour permettre aux mères de faire leurs courses en toute tranquillité. Mais dès les années 30, un changement se produit car les grands magasins transforment ces espaces de jeux - initialement découplés de l'activité marchande - en rayons présentant des produits intéressant fortement les enfants : jeux, jouets, habillement, etc. Les réflexions et prescriptions faites dans des revues professionnelles de l'époque soulignent le glissement qui s'opère peu à peu : la figure de la mère, jusque là centrale dans les achats de produits destinés aux enfants, s'efface peu à peu devant celle d'un enfant capable d'observer ses pairs et d'interpréter son environnement social, d'exprimer ses propres désirs, ce qu'il aime et ce qu'il n'aime pas, etc. La construction sociale - ou invention - du «toddler », bambin qui commence à ramper ou marcher et part explorer le monde à son initiative, apparait comme un moment rhétorique décisif qui accorde à l'enfant une capacité d'autonomie, de volonté et d'action propres qui fait qu'il échappe en partie au contrôle et aux désirs de sa mère. L'enjeu pour les distributeurs devient alors logiquement de concevoir ces espaces commerciaux qui attirent l'enfant en tenant compte de ses centres d'intérêt mais aussi des inquiétudes qui sont les siennes. De nombreux conseils apparaissent dans les revues professionnelles à la fois sur la façon d'aménager l'espace - choix des couleurs, organisation spatiale des rayons, mobilier et miroirs adaptés à la taille des enfants, etc. - qui insistent sur la nécessité par exemple de consacrer un étage entier du magasin aux produits de l'enfant avec un double profit (Cook 2004 : 117). Tout d'abord, faciliter les déambulations de la mère qui peut aisément accéder à tous les univers correspondant aux différents âges de ses enfants. Ensuite, faire en sorte qu'à la sortie des ascenseurs se trouvent les rayons destinés aux enfants les plus âgés et qu'il faille traverser tout l'étage pour accéder aux rayons concernant les bébés tels celui de la layette, car les jeunes enfants accompagnant leur mère sont alors en mesure de voir les articles proposés aux enfants plus grands et que cela entretient un effet «aspirationnel». Encore plus intéressant sur un plan symbolique, les conseils pour former le personnel au contact de cette jeune clientèle sont très révélateurs du nouveau statut accordé à l'enfant dans l'espace 
commercial $^{3}$. Il est ainsi recommandé d'éviter d'utiliser un vocabulaire trop maternant et de traiter l'enfant comme une personne capable de faire des choix sensés, le rôle du personnel de vente consistant alors à parvenir avec tact à une négociation équilibrée entre les désirs de l'enfant et les exigences de la mère (Cook 2004 : 114). Ces enseignes spécialisées ont ainsi depuis les années 30 aux Etats-Unis contribué à rendre concrète en pratique cette logique de l' «empowerment » de l'enfant en tant que consommateur, logique qui est apparue en premier lieu pour la petite fille avant de s'étendre aux univers vestimentaires des garçons.

Franck Cochoy (2008) montre, à travers une analyse fouillée des thématiques abordées entre 1922 et 1955 par la revue professionnelle "Progressive Grocer », qui s'adressait aux épiciers américains, comment l'évolution des dispositifs matériels (mobilier, innovations concernant la conservation des produits, techniques d'affichage, implantation des rayons, techniques de merchandising, etc.) a provoqué une profonde transformation du rôle de l'enfant sur le lieu de vente. Au début des années 20, l'enfant dans l'épicerie était principalement un enfant au travail puisque la revue le met en scène comme participant à des tâches annexes dans l'épicerie : nettoyage, petits trangements... En effet, à cette époque, les comptoirs sont tout simplement trop hauts, ce qui empêche les enfants de voir les produits et de prendre une part active au rituel des courses. A partir de la fin des années 30, le concept de l' «open display » se diffuse dans les épiceries américaines. De multiples innovations en termes de mobiliervitrine permettent enfin à l'enfant de voir les produits qui l'intéressent (confiseries, goûters, desserts, etc.). De ce fait, l'iconographie de la revue met en scène l'enfant comme un prescripteur attirant sa mère vers les produits qu'il désire, mais aussi comme un enfant capricieux usant de son «pester power» pour faire perdre la face à sa mère en public. En outre, à cette époque, l'enfant est également perçu comme un chapardeur en puissance et des systèmes de tourniquets intégrant un tablier métallique sont proposés pour équiper l'entrée des magasins afin d'empêcher l'enfant de ramper et de s'échapper en emportant des produits. Dès le milieu des années 40, le développement du libre service est rendu possible par des innovations en termes de conservation et de présentation des produits (appertisation, cellophane, etc.) et la généralisation de l'usage des caddies. En lui offrant de manœuvrer un caddie miniaturisé, l'épicier va inciter l'enfant à imiter directement le consommateur adulte et à incorporer l'ensemble de sa gestuelle ordinaire: circuler dans les rayons, choisir des produits, passer à la caisse, etc. Dès lors, les techniques de merchandising évoluent et prennent en compte l'enfant en plaçant les produits qui le concernent à la bonne hauteur dans les rayons, en lui présentant une sélection de produits dans les têtes de gondoles en forme de petites maisons ou de cabanes, en mettant en œuvre des animations ludiques pour Halloween, et en créant des univers totalement dédiés aux produits qui lui sont destinés.

Bien que ces différents travaux ne soient pas centrés sur les mêmes objets de recherche, ils se rejoignent pour montrer à quel point les innovations liées aux techniques de distribution à la fois exploitent et renforcent certaines évolutions conceptuelles et institutionnelles à propos de l'enfant, de son éducation, de son aptitude à décider et à exprimer ses choix, qui émergent dans la société et son légitimées par des discours scientifiques - émanant de recherches en psychologie, en sociologie ou des sciences de l'éducation - ou des discours politiques. L'évolution des techniques marketing employées par la distribution ont ainsi conduit à transformer le rôle et le statut de l'enfant au cœur même du magasin. En ce qui concerne le choix des produits sur le lieu de vente, l'enfant est ainsi passé d'une participation indirecte et périphérique - comme destinataire du produit acheté par sa mère principalement - à une participation centrale et active - comme décideur sur les catégories de produits dans lesquels

\footnotetext{
${ }^{3}$ Interview d'un manager de la chaîne L.S. Ayers \& Co, rapportée dans la Revue Dry Goods Economics du 19 Novembre 1921. Référence donnée par D. Cook (2004) page 174.
} 
il est fortement impliqué... L'émergence relativement récente de magasins spécialisés sur différents domaines de la vie de l'enfant, à la fois poursuit, accompagne et suscite un double mouvement de société plus large et plus profond : l'institutionnalisation de l'enfant comme un acteur à part entière de sa propre consommation et une logique de centration sur l'enfant dans différentes sphères de la société contemporaine qui dépassent le simple environnement commercial (Gavarini 2004).

\section{2 - Les techniques de distribution comme médiateurs de la participation de l'enfant aux activités de consommation}

En s'affirmant distributeurs spécialistes de l'enfant et en concevant des magasins adaptés à ce client particulier, les enseignes prennent acte de - mais également renforcent - l'autonomie de l'enfant, sa capacité à tenir pleinement son rôle de jeune consommateur dans la société contemporaine. Toutefois, de l'ensemble des développements précédents, il ressort que l'enfant dans l'espace commercial dispose d'une autonomie très relative et développe toujours en réalité une activité conjointe de consommation (La Ville \& Tartas 2005). En effet, l'enfant se trouve toujours accompagné par ses parents sur le lieu de vente, du moins au début; ensuite, il peut se trouver accompagné dans un magasin par un enfant plus expérimenté sur la catégorie de produits distribués, comme son grand frère ou un ami ; et finalement, même si l'enfant se trouve apparemment tout seul devant les linéaires d'un espace commercial qui lui est dédié, il va faire référence à un certain nombre d'éléments qui lui ont été préalablement inculqués par sa famille ou ses pairs pour faire ses choix. Avant même d'arpenter l'espace commercial, l'enfant a déjà intégré une bonne part des acquis culturels liés à la consommation de masse grâce aux produits que ses parents, sa fratrie, ses grands-parents rapportent au foyer. En effet, l'enfant n'a pas à réinventer les concepts de marque, de packaging ou de linéaire, car tous ces éléments font partie de notre culture contemporaine de consommateurs.

Dans cette perspective, la relation de l'enfant à l'espace commercial n'est pas directe mais médiatisée par un ensemble d'outils culturels : des notions conceptuelles sur les produits, les prix, la qualité, les marques, les logos, etc., mais aussi des éléments matériels tels le mobilier, les linéaires, l'agencement spatial et sensoriel du point de vente. C'est à travers l'ensemble de ces catégories symboliques et matérielles que l'enfant peut aborder le sens de l'organisation de l'espace commercial et du rôle qu'il peut éventuellement y jouer. Il convient alors de bien cerner les médiateurs symboliques et matériels qui rendent possible la participation de l'enfant à l'acte de consommation sur le lieu de vente. Ce qui suppose également de comprendre quels fondements institutionnels sont mobilisés pour légitimer la participation de l'enfant à l'activité sur le lieu de vente, comme nous allons l'illustrer avec le cas de l'entreprise suivante.

\section{Encadré $\mathbf{N}^{\circ} 2$ : Le concept KIDFRESH}

\section{Projet de création de valeur}

Cette société ${ }^{4} \mathrm{~s}^{\prime} e s t$ donné pour mission de pallier l'absence de cantines dans la plupart des établissements scolaires aux Etats-Unis, situation qui conduit les parents pressés à composer un déjeuner avec des sodas et divers sachets de produits faciles à grignoter, menu quotidien présentant de trop fortes teneurs en sucre, sel et produits gras. Les fondateurs ont en outre constaté que 25 à $30 \%$ d'enfants américains ne mangent quasiment jamais de légumes ou de fruits frais. La question de ces créateurs d'entreprise était de savoir s'ils pouvaient faire

\footnotetext{
${ }^{4}$ Site de la société kidfresh : http://www.kidfresh.com/
} 
quelque chose pour changer cette situation qui participe directement à l'extension de la pandémie d'obésité aux Etats-Unis. Comment faire pour que les enfants apprécient la qualité des produits frais, qu'ils se reconnaissent dans des aliments sains, les réclament et se les approprient comme faisant partie de leur propre univers ? A l'ouverture du premier magasin à New York en Juin 2007, cette société proposait deux types de prestations :

\section{Un magasin alimentaire incluant un « Kidfresh bar » pour les enfants}

Les enfants disposent d'une entrée propre dans le magasin (interdite aux parents) et peuvent utiliser des caddies à leur taille. L'agencement du magasin comprend classiquement des linéaires présentant des produits alimentaires ayant fait l'objet d'une sélection très stricte : produits naturels et biologiques, sans conservateur, sans arôme artificiel et sans graisses hydrogénées, mis au point par des producteurs de taille moyenne, voire parfois artisanale. La sobriété des packagings est destinée à simplifier la compréhension du message par les enfants. Une catégorisation originale est mise en place (baby, mini, junior et kids) avec la préoccupation d'indiquer les apports nutritionnels qui conviennent à chaque tranche d'âge. Cette information facile à comprendre constitue un élément de réassurance pour les parents. En outre, l'offre proposée ne comprend pas que des aliments et inclut un ensemble de produits éducatifs à propos de l'alimentation : des jeux, des magazines éducatifs sur l'alimentation.

Un espace original côtoie les linéaires : c'est le « Kidfresh Bar » à proximité de la caisse. Cet espace regroupe une machine où l'enfant peut choisir - et se faire conseiller sur - les produits frais avec lesquels les cuisiniers vont lui confectionner le sandwich qu'il aura lui-même inventé. L'enfant va en outre pouvoir choisir la forme de son sandwich découpé dans du pain de mie : soit une main, soit un visage souriant. Ensuite, l'enfant va manger le sandwich qu'il aura confectionné dans un espace dédié comprenant un petit lavabo pour se laver les mains. Pendant que les enfants mangent et s'amusent, ils peuvent voir les cuisiniers en train de préparer la nourriture à travers une vitre transparente.

Tout est mis en scène pour que l'enfant soir actif en ce qui concerne le choix des ingrédients et l'hygiène. En outre, une dimension ludique est omniprésente : sur les packagings par la présence d'animaux ou de dessins amusants qui font référence à l'univers enfantin et à ses plaisirs ; mais aussi dans la façon d'impliquer l'enfant dans la préparation de son repas composé de produits frais. L'entreprise refuse de mobiliser des personnages sous licence en faisant le pari que cela permettra de nouer une relation spécifique avec l'enfant, différente de celle des grandes marques alimentaires classiques, qui utilisent des personnages issus de dessins animés ou de l'édition pour créer un lien affectif entre la marque et l'enfant.

\section{Un ensemble de services destinés aux parents}

Au-delà du point de vente lui-même, la société a mis en place un service destiné aux parents pressés : la fourniture d'une «lunch-box» contenant des produits issus de l'agriculture biologique (un sandwich élaboré avec des légumes frais ayant une forme amusante, un dessert composé de fruits frais et une bouteille d'eau) qui est directement livrée dans l'école fréquentée par l'enfant. Par ailleurs, les projets d'implantation de points de vente sont relativement originaux, avec des magasins dans les grandes villes, mais aussi des distributeurs dans les aéroports et dans des lieux où les parents et les enfants passent un moment ensemble.

Source: extraits de la plaquette de la société KidFresh et d'une interview de Monsieur Matt Cohen, fondateur, que nous avons réalisée lors de la préparation du $\mathrm{X}^{\circ}$ Colloque Etienne THIL en Octobre 2007.

En pratiquant un «marketing sensoriel » qui s'adresse directement à l'enfant et en apportant une solution institutionnelle qui réaffirme le rôle éducatif des parents en matière 
d'alimentation, cette entreprise propose de transformer la participation de l'enfant à l'activité conjointe de consommation alimentaire, à la fois dans le cadre scolaire mais aussi dans des moments ordinaires dans la vie de l'enfant, avec une visée clairement éducative. Le concept proposé par «Kidfresh» renforce les parents dans leur rôle de parents responsables, suffisamment aimants ${ }^{5}$ (Winnicott 1975), car il leur propose des supports matériels et ludiques pour éduquer leur enfant à une alimentation saine. Le fait de fréquenter cette enseigne permet aux parents d'affirmer leurs compétences éducatives et leur système de valeurs (La Ville \& Tartas 2008).

Ainsi, innover dans les réseaux de distribution dédiées à l'enfant suppose de trouver comment aménager la participation conjointe de l'enfant et de l'adulte (parent - grands-parents éducateur) à l'activité de consommation. Les médiateurs matériels mobilisés pour concevoir et organiser le point de vente ainsi que les médiateurs symboliques pour exposer le bénéfice apporté par le produit et la prestation de service doivent s'appuyer sur un substrat institutionnel permettant à la fois de légitimer la participation directe de l'enfant à l'activité de consommation tout en y impliquant le parent et en le valorisant dans son rôle éducatif. Ce qui suppose pour l'enseigne de mettre en œuvre de façon explicite une politique de responsabilité sociale de l'enseigne pour aller au-delà des discours convenus, pour annoncer les valeurs qu'elle pratique et pour mettre en place des indicateurs de progrès transparents, contrôlables, susceptibles de créer de la confiance vis-à-vis des adultes ayant la responsabilité de l'éducation des enfants. Sur ce registre, la société ID Group, spécialiste de mode enfantine qui détient plusieurs enseignes comme «Okaïdi », «Obaïby» ou encore «Jacadi », qui va prochainement atteindre les 1000 points de ventes au plan international, a mis en place un système appelé « ̈̈dway: entreprendre pour que le monde progresse au service de l'enfant qui grandit ». Les fondateurs affirment les valeurs du groupe ${ }^{6}$ : «La démarche (du groupe), c'est les droits de l'enfant, c'est un cri de joie et de confiance dans la vie. C'est une démarche collective. C'est en gros, quelle planète veut-on laisser à nos enfants ? (...) Nous avons une démarche RESSEME, c'est-à-dire de responsabilité entrepreneuriale, sociale, sociétale et environnementale d'une marque. Elle fixe l'ensemble des valeurs et responsabilités que nous voulons partager et réussir avec les parties prenantes de notre projet : enfants, parents, grandsparents, collaborateurs et leurs familles, fournisseurs, partenaires collectivités, institutionnels, associations locales, ONG, actionnaires... » Elle se décline de la façon suivante :

\section{Encadré n ${ }^{\circ} 3$ : La conception élargie de la Responsabilité Sociale de ID Group}

Entrepreneuriale : c'est notre responsabilité économique de décideur, chargé de développer et de pérenniser l'entreprise, et

Sociale : c'est notre responsabilité humaine d'accompagnement pédagogique pour que chacun s'épanouisse et progresse dans son métier, et

Sociétale : c'est notre responsabilité dans la cité à agir et à faciliter le progrès de tous les acteurs qui sont parties prenantes de notre projet, et

Environnementale : c'est notre responsabilité écologique pour le respect du développement durable de la planète que nous transmettrons à nos enfants, des

Marques : c'est notre responsabilité politique (notre mission) «Entreprendre pour que le monde progresse au service de l'enfant qui grandit », pour les

Enfants : c'est notre responsabilité de service pour répondre à tous les besoins pratiques, esthétiques et éthiques de chaque enfant dans le monde.

\footnotetext{
${ }^{5}$ « La mère (qui n'est pas forcément la propre mère de l'enfant) suffisamment bonne est celle qui s'adapte activement aux besoins de l'enfant. » (Winnicott, $1975: 19)$

${ }^{6}$ Idway Les engagements de progrès d'Idgroup - Magazine du groupe - Edition Juin 2007, page 3.
} 
Il s'agit d'un projet ambitieux et volontariste - car le groupe n'est pas coté en bourse d'affirmation des valeurs qui orientent son travail afin d'établir une relation de confiance à la fois avec les adultes et les enfants pour promouvoir une consommation responsable de produits de mode et d'habillement.

\section{Conclusion : Enrichir la relation entre l'enfant et l'espace commercial au- delà du point de vente}

En guise de conclusion très provisoire, nous voudrions ouvrir cet article sur la nécessité d'explorer la façon dont les enseignes de distribution, généralistes et spécialistes de l'enfant, utilisent les nouveaux médias pour valoriser leur marque et leurs produits au cœur même des pratiques ludiques et culturelles des enfants. Les enseignes ont accès à la publicité télévisée, et la directive Télévision sans frontières va permettre d'assouplir les modalités d'intégration de la publicité dans différents types de supports culturels. La pratique de 1' «advergaming », consistant à offrir à l'enfant une activité ludique centrée sur les attributs d'une marque ou de produits, a été dénoncée par une étude de la Kaiser Family Fondation (Moore 2006) qui montre comment les sites de marques établissent une relation très intime avec l'enfant absorbé et immergé dans le jeu pour insérer leurs produits dans les univers culturels de l'enfant. Or, l'année 2007 a connu un renversement majeur dans les pratiques de loisirs des enfants : le temps passé à regarder la télévision a été détrôné au profit du Web, et en particulier du Web 2.0. Cela signifie que les enfants ne vont pas uniquement consommer des émissions ou des jeux, mais de plus en plus les produire eux-mêmes et les diffuser sur des sites communautaires. Les enfants vont ainsi devenir des co-producteurs de contenu culturel et les enjeux relatifs à la prise en compte de ces nouvelles pratiques culturelles devront être intégrés pour valoriser les enseignes. C'est un réel défi qui se pose aux enseignes qui souhaitent utiliser l'interactivité qu'offre le web pour s'adresser à l'enfant et établir une relation créative avec lui, au-delà du point de vente... mais sans pour autant confisquer la créativité des enfants au profit exclusif de la marque ou de l'enseigne.

\section{Références bibliographiques}

Benoun M. et Héliès-Hassid M. L. (2003), Distribution : acteurs et stratégies, $3^{\circ}$ éd., Paris, Economica. $415 \mathrm{p}$.

Cliquet G., Fady A. et Basset G. (2002), Management de la distribution, Paris, Dunod, 340 p. Cochoy F. (2008), « Hansel and Gretel at the grocery store: Progressive Grocer and the little American consumers (1929-1959) », Journal of Cultural Economy, (à paraître).

Cook D.T. (2004), The commodification of childhood: the children's clothing industry and the rise of the child consumer, Durham, London, Duke University Press, 211p. Voir en particulier le chapitre 5: «Reconfiguring girlhood: age grading, size ranges and aspirational merchandising in the 1930's $»$.

Cowles S. (2002), "Creating the ultimate baby and toddler-friendly environment », International Journal of Advertising and Marketing to Children, 3( 3), April / June, pp. 41- 8. Daucé B. et Rieunier S. (2002), «Le marketing sensoriel du point de vente », Recherche et Applications en Marketing, 17(4), pp. 45-65.

De La Ville V.I. et Tartas V. (2008), « Transformer la participation de l'enfant aux activités de consommation alimentaire », in Schaal B. (Ed.), Revue Enfance, Numéro thématique 
«L'enfant face aux aliments : de préférences en programmations », Presses Universitaires de France (à paraître).

De La Ville V.I. et Tartas V. (2005), «L'activité de consommation enfantine et ses médiateurs », in De La Ville V.I. (Coord.), L'enfant consommateur: variations interdisciplinaires sur l'enfant et le marché, Paris, Vuibert, pp. 73-88.

Fielder A., Gardner W., Nairn A. et Pitt J. (2007), Fair Game? Assessing commercial activity on children's favourite websites and online environments, Report for the National Consumer Council, London.

Gallouj K. (2007), Innover dans la grande distribution, Editions De Boeck Université, Bruxelles.

Gavarini L. (2004), La passion de l'enfant : filiation, procréation et éducation à l'aube du XXIe siècle, Paris, Hachette littératures.

Héliès-Hassid M. L. (2000), « Au Bonheur des dames ou la leçon de commerce moderne de M. Zola », Décisions Marketing, 20, Mai-Août, pp. 35-46.

Héliès-Hassid M. L. (2007), «Les enfants et la distribution : kids retailing », in Brée J. (Coord.), Kids Marketing, Colombelle, Editions EMS, pp. 347-392.

Jallais J., Orsoni J. et Fady A. (1987), Le Marketing de la distribution application au point de vente, Paris, Vuibert, $418 \mathrm{p}$.

Moore E. S. (2006), It's Child's Play: Advergaming and the Online Marketing of Food to Children, A Kaiser Family Foundation Report, July, 51 p.

Mouton D. et Paris G. (2007), Pratique du merchandising, $2^{\circ}$ édition, Editions Dunod, Paris.

Szymanski M. (2002), « Marketing toys by developmental stages », International Journal of Advertising and Marketing to Children, 3(2), January - March, pp. 25-32.

Volle P. (2000), Etudes et recherches sur la distribution, Paris, Economica.

Weiner N. (2004), « How to engage with today's kids. Successful store design: a case study featuring Lego brand stores », Young Consumers, 4(4), Quarter 3, pp. 46-52.

Winnicott D. (1975), Jeu et réalité : l'espace potentiel, Gallimard, Paris. 